\title{
Analysis and Application of Infrared Phase Image
}

\author{
Jinyu Zhang \\ Xi'an Research Institute of High-tech, Xi'an, 710025, P. R. China
}

\begin{abstract}
Infrared sequence image processing is widely used in engineering, but the research of infrared phase processing is rare. In this paper, a method of generating thermal wave phase image based on infrared phase locked-in, phase difference processing and its application in coatings defect detection and recognition are presented. The experimental results show that the method is effective and practical.
\end{abstract}

Keywords-image processing; phase difference; thermal wave image processing; defect recognition

\section{INTRODUCTION}

Infrared image processing is an important part of image processing, which is widely used in military, aerospace, petrochemical, construction, electric power, medicine and other fields. The key is to process the radiation intensity of infrared thermal image or image sequence, to effectively restrain the adverse influence such as infrared image noise, uneven heating, infrared radiation deviation, surface morphology and other factors, to extract target information clearly [1]. It is found that the phase information of infrared thermography is not related to the radiation rate and morphology of the detected object, and it can obtain better detection results [2].

At present, a variety of estimation methods have been proposed for phase difference estimation [3-7]. Circuit based zero detection method calculates phase difference according to two signal zero crossing time. The calculation speed is fast, but the cost of hardware is high and the anti-interference ability is weak; Digital correlation method uses correlation function to estimate the phase difference of two signals, has good random noise suppression ability, but by the harmonic influence and sampling; High order spectrum algorithm estimates the phase difference based on complex high order spectrum with large amount of calculation; Hilbert transform method first need 90 degree phase shift of the signal, then calculate the phase difference by function between two signals before and after phase, can dynamically estimate the time-varying phase difference, but is susceptible to harmonic interference. The phase difference estimation of DFT is obtained by the phase subtraction of the maximum spectral line of the signal discrete spectrum, and the estimation accuracy is relatively high. The FFT algorithm can be used in a wide range. However, the spectrum leakage during the DFT calculation will affect the accuracy of phase difference estimation [5-6].

In this paper, a method of generating thermal wave phase image based on infrared phase locked-in, phase difference processing and its application in coating defect detection and recognition are presented.

\section{Phase Image Detection}

The principle of phase detection is shown in figure $\mathrm{I}$. Heating equipment makes use of sine wave energy output to heat on the surface of material. The thermal wave will affect the change rate and delay of surface temperature in the process of spreading inside the material if it encountered defects or uneven thickness or other material. The surface temperature is recorded by a thermography in real time, and the phase processing algorithm is applied to temperature-time sequences, to calculate the phase and phase difference, and to detect and identify the defect location, size and other relevant information.
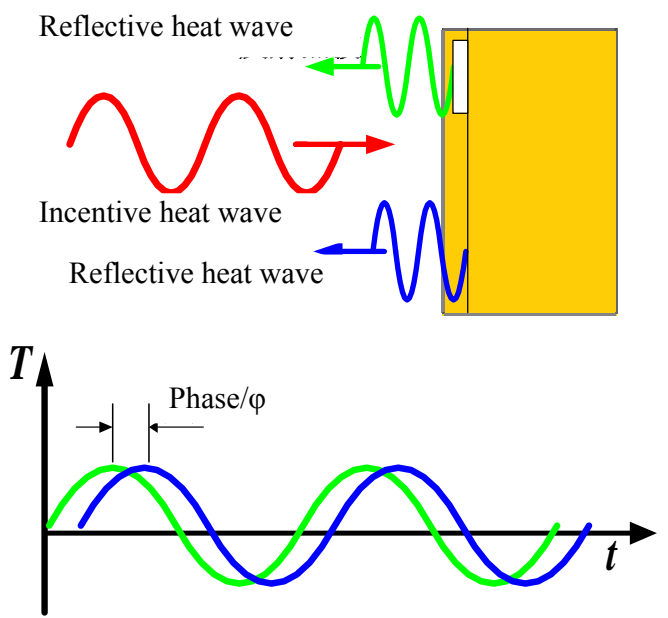

FIGURE I. PRINCIPLE OF COATING DEFECT DETECTION BY PHASE

If the temperature of a point in the infrared image is $T(n)$, and the sequence length is $\mathrm{N}$, then the discrete Fourier transform (DFT):

$$
F(k)=\operatorname{DFT}[T(n)]=\sum_{n=0}^{N-1} T(n) e^{-j 2 \pi n k / N}=\sum_{n=0}^{N-1} T(n) W_{N}^{n k}
$$

Where $W_{N} \mathrm{~N}$ point DFT core function, $W_{N}=e^{-j 2 \pi / N}$

When the length of temperature signal is $\mathrm{N}$, and the sampling rate of thermal imager is $f_{\mathrm{s}}$, the fundamental frequency of FFT is $f_{1}=f_{\mathrm{s}} / N$, and the amplitude and phase of the harmonic component of the frequency $k f_{1}$ is. 


$$
\begin{aligned}
& A(k)=|F(k)| \\
& \varphi(k)=\tan ^{-1} \frac{I_{m}[F(k)]}{R_{e}[F(k)]}
\end{aligned}
$$

FFT transform of the entire temperature signal are calculated, the noise of a single point will not have a significant impact on the calculation results, so the FFT algorithm has good effect of noise signal processing. At the same time, a full period sampling is used in order to ensure the accuracy of phase detection in this method.

\section{STYLING EXPERIMENT AND ANALYSIS}

\section{A. Experimental Specimen}

The adhesion problem between the coatings and substrate is a kind of common defects in the practical application, for example, the deboned defect of surface coatings of solid rocket motor glass fiber winding composite shells and the substrate, and the coatings on metal substrate of most the weapon system, are very easy to produce debonding defects. Therefore, the test specimens are made of two kinds of materials: aluminum and glass fiber. The coatings is made of phenolic black antirust paint, and the defects are simulated by PTFE interlayer, and the sample is made of two pieces. The specimens are shown in Figure II and figure III.

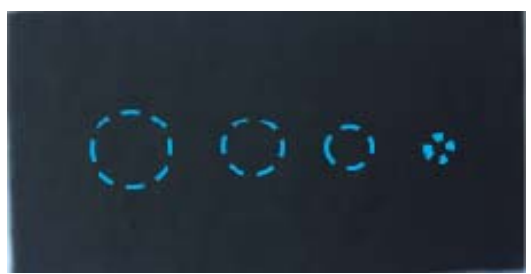

(a) Specimen face

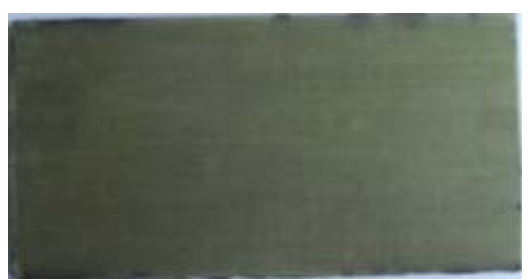

(b) Specimen back

FIGURE II. DEBONDING DEFECT SPECIMENS OF GLASS FIBER MATRIX COATINGS

The fiber glass specimen specifications: length $200 \mathrm{~mm}$, width $100 \mathrm{~mm}$, substrate thickness $5 \mathrm{~mm}$, coating thickness $2 \mathrm{~mm}$, between the coatings and the substrate with 4 round PTFE layer to simulate debonding defects, debonding defect diameter is $30 \mathrm{~mm}, 25 \mathrm{~mm}, 16 \mathrm{~mm}$ and $8 \mathrm{~mm}$.

Aluminum sample size: the substrate length $200 \mathrm{~mm}$, width $80 \mathrm{~mm}$, thickness $5 \mathrm{~mm}$, coatings thickness of $2 \mathrm{~mm}$, defects in 3 square PTFE sandwich simulation, defect side length is $30 \mathrm{~mm}, 20 \mathrm{~mm}$ and $10 \mathrm{~mm}$.

\section{B. Experimental Results}

Using two PAR64 series halogen lamp as the thermal excitation light source, each halogen lamp power is $1 \mathrm{KW}$, the light source intensity changes with sine law, the highest frequency is $0.2 \mathrm{~Hz}$. The lowest is $0.002 \mathrm{~Hz}$. The experiment captured three cycles of temperature series infrared images, each cycle fixed 100 frames. In order to make the results more accurate, the latter two cycle temperature sequence were chosen, namely the 101 300 frame.

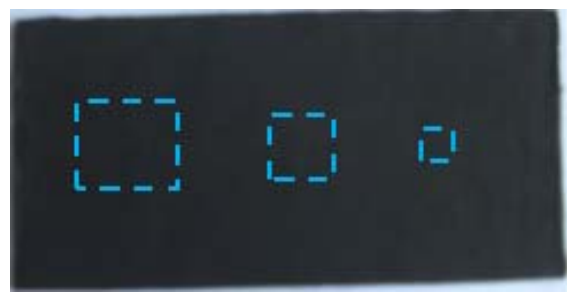

(a) Specimen face

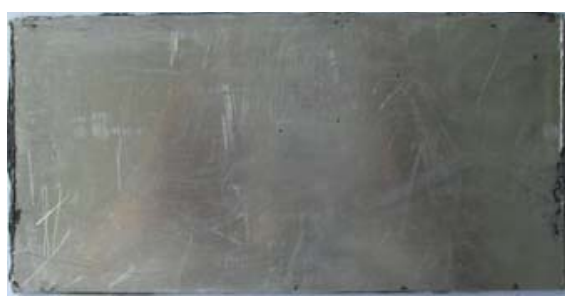

(b) Specimen back

\section{FIGURE III. COATINGS DEFECT OF ALUMINUM MATRIX}

At the center of the four defects and a non-defect point on the glass fiber specimens, a total of 5 points are selected. Figure IV is the temperature curve of the 5 points. It can be seen from the chart, the temperatures of the 5 point rise along the sinusoidal variation. The non-defect point temperature is lowest in theory. The bigger the defect size is, the higher the defect center temperature is. But in the figure, the center temperature of the defect of diameter $16 \mathrm{~mm}$ is the highest, followed by $12 \mathrm{~mm}, 25 \mathrm{~mm}$ and $8 \mathrm{~mm}$. This is due to the thermal excitation light source has two halogen lamps, in which thermal excitation caused uneven heating on the surface of coatings by operation. The middle part of coatings surface get more heat, both sides get less, so the middle is high temperature. 


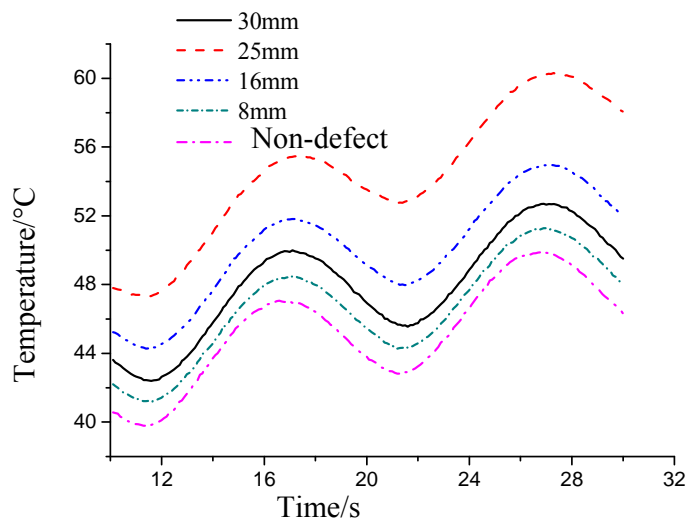

FIGURE IV. TEMPERATURE CURVES OF GLASS FIBER SPECIMENS

Take 4 points in the three defect centers and defect free parts of aluminum specimens. Figure $\mathrm{V}$ is the temperature change curve. As can be seen from the figure, the temperature change is not consistent with the theory, which is the same as the glass fiber specimen surface heating, on the surface of the aluminum specimen, two halogen lamps heating can't be uniform. Although the diameter of $20 \mathrm{~mm}$ and $10 \mathrm{~mm}$ defect center temperature is very similar, but it can be clearly seen that the temperature curves of two points is different before and after.

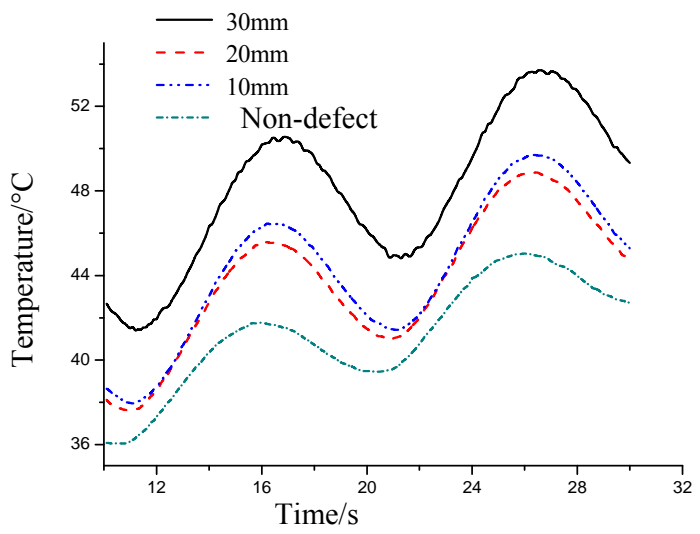

FIGURE V. TEMPERATURE CURVES OF ALUMINUM SPECIMEN

Randomly selected 6 points at far away from the defect and the defect free region in the aluminum specimens, the each point temperature curve is as shown in Figure VI. Due to nonuniform heating excitation, there are some differences between the temperatures of each point, but the temperature difference only existed in different amplitude. It can be seen from the chart, each point of the curve there is no about phase shift. This shows that although the amplitude of temperature is different, but the phase of each point is the same. It also confirmed a significant advantage of lock-in method: not affected by the uneven heating of the light source.

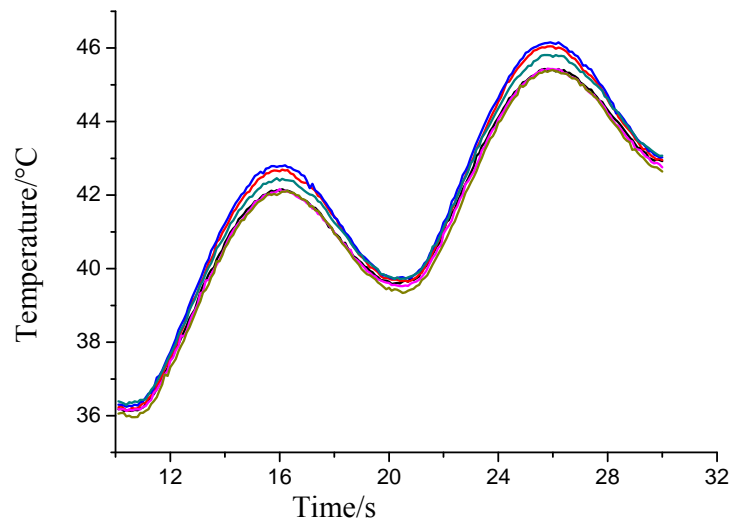

FIGURE VI. TEMPERATURE VARIATION CURVES OF ALUMINUM SPECIMEN WITHOUT DEFECT

Based on the principle of thermal wave detection, the thermal excitation of the optimized frequency of $0.01 \mathrm{~Hz}$ is selected to heat the surface of the coatings, and the phase amplitude is represented by gray scale. The phase image of the two samples is shown in figure VII.

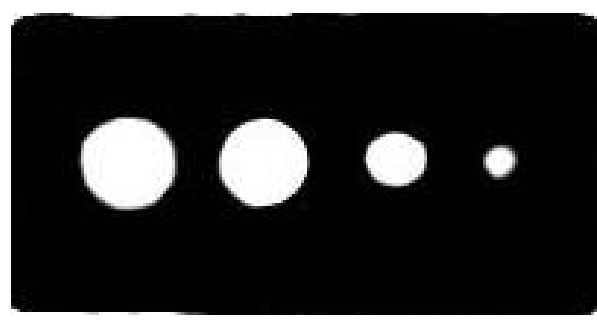

(a)



(b)

FIGURE VII. PHASE IMAGES OF GLASS FIBER SPECIMEN (A) AND ALUMINUM SPECIMEN (B)

\section{APPLICATION}

Take the horizontal lines along the center of the two specimens, extract the phase of temperature sequence of the pixels on the line, and do the first order difference, as shown in Figure VIII and figure IX. It can be seen that two phase difference curve contains multiple peaks and valleys from the figures. Each defective pixel range consists of a peak and a trough. Peak value occurs at the left of defects, and valley occurs at the right. In addition to difference value corresponds to four defects; phase difference also contains some small amplitude changes. This is due to the noise of specimen preparation and image capturing. The phase difference obviously changes at the left and right sides of specimen, 
because these pixels are so close to the edge of the specimen. Because each pixel has a fixed proportion relation with the actual size, the size of the defect can be calculated according to the pixels between the left and right edges of the defect.

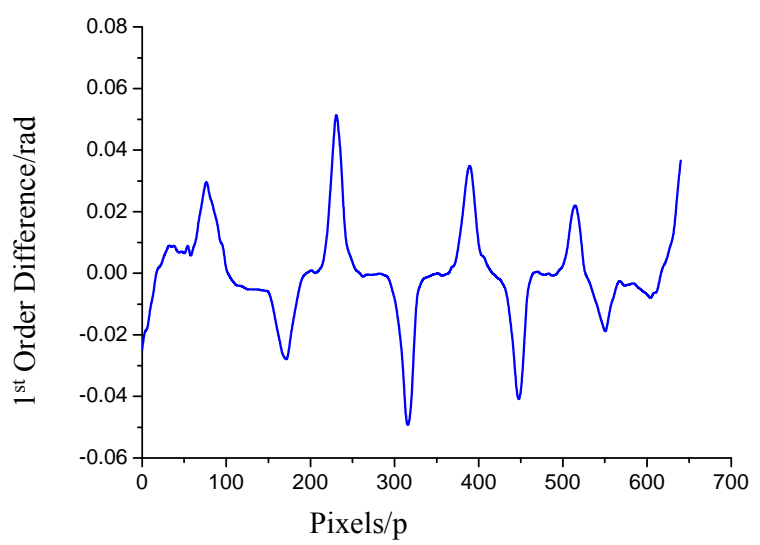

FIGURE VIII. FPHASE DIFFERENCE CURVE OF GLASS FIBER SPECIMEN

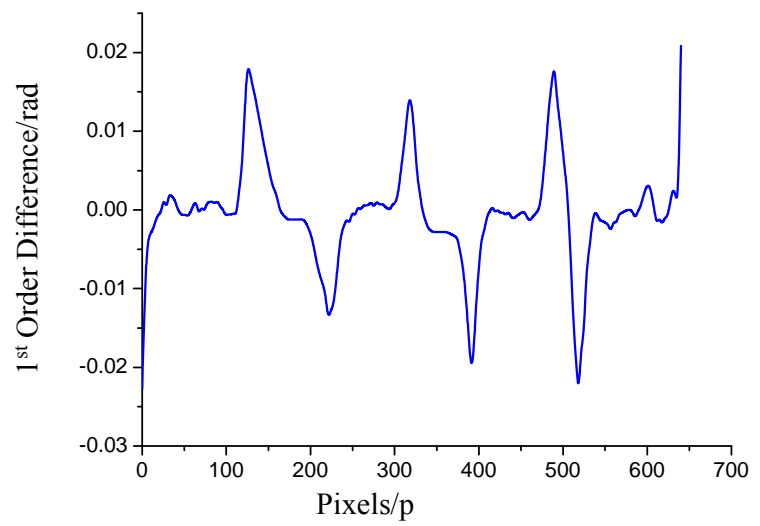

FIGURE IX. PHASE DIFFERENCE CURVE OF ALUMINUM SPECIMEN

By calculation, the defect sizes of the two specimens are shown in Table I, respectively:

TABLE I. DEFECT SIZE IDENTIFICATION

\begin{tabular}{|c|c|c|c|}
\hline Type & $\begin{array}{c}\text { Real } \\
\text { size/mm }\end{array}$ & $\begin{array}{c}\text { Calculate } \\
\text { d size } / \mathrm{mm}\end{array}$ & Error \\
\hline \multirow{4}{*}{$\begin{array}{c}\text { Glass } \\
\text { fiber } \\
\text { specimen }\end{array}$} & 30 & 30.625 & $2.08 \%$ \\
\cline { 2 - 4 } & 20 & 20.938 & $4.69 \%$ \\
\cline { 2 - 4 } & 8 & 17.5 & $5.47 \%$ \\
\hline \multirow{3}{*}{$\begin{array}{c}\text { aluminu } \\
\text { m specimen }\end{array}$} & 30 & 8.94 & $9.38 \%$ \\
\cline { 2 - 4 } & 16 & 21.003 & $3.15 \%$ \\
\cline { 2 - 4 } & 10 & 10.916 & $9.02 \%$ \\
\hline
\end{tabular}

The maximum error is $9.38 \%$; the minimum is $2.08 \%$, basically meet engineering requirements. It can be found that the smaller the defect size is, the larger the error is. The smaller the defect size is, the larger the proportion of the defect size is in the range of the phase change, and the larger the error percentage is when the defect edge position is determined.

\section{CONCLUSION}

This article investigates the phase image detection and its application in defect recognition. Infrared imaging processing results show that the phase image is relatively clean, less interference, and have strong expression ability. The differential processing can effectively identify targets. More in-depth research is valuable.

\section{ACKNOWLEDGMENT}

This work was supported by the Chinese National Science Foundation under Contract No. 51275518.

\section{REFERENCES}

[1] Jin-Yu Zhang, Wei Zhang, Zheng-Wei Yang, A novel algorithm for fast compression and reconstruction of infrared thermographic sequence based on image segmentation, Infrared Physics \& Technology, 67(2014) 296-305

[2] J.Y. Zhang, X.B. Meng, and Y.C. Ma, "A new measurement method of coatings thickness based on lock-in thermography," Infrared Physics \& Technology, vol. 76, pp. 655-660, 2016.

[3] R. Shrestha, J. Park, and W. Kim, "Application of thermal wave imaging and phase shifting method for defect detection in Stainless steel," Infrared Physics \& Technology, vol. 76, pp.676-683, 2016.

[4] R. Waugh, J. Dulieu-Barton, and S. Quinn, "Modeling and evaluation of pulsed and pulse phase thermography through application of composite and metallic case studies," NDT \& E International, vol. 66, pp. 52-66, 2014.

[5] Qi Guo-qing. Accuracy analysis and comparison of some FFT-based frequency estimators[J]. Journal of Vibration Engineering, 2006, 19(1): $86-92$

[6] Li Hui, Wang Yan-fei, and Li Jiong. On the parameter estimation of sinusoidal signal[J]. Journal of Electronics \& Information Technology, 2007, 29(11): 2653-2656.

[7] Shu Ting, Zhao Wei-hong, and Lu Jin-hui. Performance analysis for fas frequency estimation with short sequences[J]. Systems Engineering and Electronics, 2006, 28(10): 1509-1512. 